\title{
Teor de colesterol e composição centesimal de algumas espécies de peixes do estado do Ceará
}

\author{
Cholesterol content and proximate composition of some fish species from the Ceará state
}

\author{
Francisco Charles Barros CAULA ${ }^{1}$, Maria Petronília de OLIVEIRA², Everardo Lima MAIA ${ }^{2 *}$
}

\begin{abstract}
Resumo
O presente estudo tem como objetivo determinar o teor de colesterol total em amostras de pescado de água marinha e doce. As amostras analisadas foram o pargo marinho, Lutjanus purpureus, e os seguintes peixes de água doce: tilápia do Nilo, Oreochromis niloticus, curimatã, Prochilodus cearensis e a sardinha, Triportheus angulatus, adquiridas em pontos comerciais (frigoríficos, feira de pescado da praia de Mucuripe e feira-livre suburbana) de Fortaleza - Ceará. Além da determinação do teor de colesterol, também foram avaliados a composição química centesimal e o valor calórico das amostras. O teor médio de colesterol (mg.100 g-1) apresentado foi 33,5 para o pargo, 28,4 para a tilápia, 93,8 para o curimatã e 61,2 para a sardinha. Em relação à composição centesimal, os seguintes teores médios foram obtidos, respectivamente, para pargo, tilápia, curimatã e sardinha: 80,$7 ; 80,2 ; 76,4$ e 77,2\% de umidade; 18,$4 ; 17,7 ; 18,7$ e $17,6 \%$ de proteína total; 1,$0 ; 1,2 ; 3,2$ e 4,6\% de lipídio total; 0,$7 ; 0,8 ; 0,8$ e 1,0\% de cinza, e 0,5; 0,6; 2,0 e 0,3\% de carboidratos. O valor energético médio ( $\mathrm{kcal} 100$ g $\left.\mathrm{g}^{-1}\right)$ foi de 83,6 para o pargo, 83,7 para a tilápia, 108,4 para o curimatã e de 111,8 para a sardinha.
\end{abstract}

Palavras-chave: peixe marinho; peixe de água doce; colesterol; valor nutritivo; valor energético.

\begin{abstract}
The purpose of this work is to determine the total cholesterol content and proximate composition of the commercial fish from the Ceará State, Brazil. The species were the marine bream fish Lutjanus purpureus and three freshwater fish, tilapia, Oreochromis niloticus, "curimatã", Prochilodus cearensis, and sardine, Triportheus angulatus, from the open market (Mucuripe and around the city) and from a supermarket in Fortaleza. The content of cholesterol, proximate composition, and the caloric value of the samples were determined. The average value of cholesterol (mg.100 g ${ }^{-1}$ ) was 33.5 in the red sea bream; 28,4 in the common tilápia; 93.8 in the "curimatã" and 61.2 in the sardine. Regarding the proximate composition, the following average values were obtained: $80.7 ; 80.2 ; 76.4$, and $77.2 \%$ of humidity, 18.4; 17.7; 18.7 , and $17.6 \%$ of the total protein; $1.0 ; 1.2 ; 3.2$; and $4.6 \%$ of total lipids; $0.7 ; 0.8 ; 0,8$; and $1.0 \%$ of the ash, and $0.5 ; 0.6,2.0$; and $0.3 \%$ of carbohydrates, respectively for the red sea bream, tilapia, "curimatã" and sardine. The energetic average value (kcal.100 g $\left.\mathrm{g}^{-1}\right)$ was 83.7 for the red sea bream, 83.6 for tilapia; 108.4 for "curimatã", and 111.8 for sardine.

Keywords: marine fish; freshwater fish; cholesterol; nutritive value; caloric value.
\end{abstract}

\section{Introdução}

O colesterol é um precursor de hormônios e vitaminas necessários para os processos vitais de reprodução e manutenção da saúde dos animais. Cerca de $70 \%$ do total de colesterol é produzido pelo próprio organismo e o restante é originário da dieta, principalmente de origem animal (CORMILLOT, 2002). Todavia, o consumo excessivo de alimentos considerados ricos em colesterol ou contendo ácidos graxos precursores de colesterol (14:0 e 16:0) provoca um desequilíbrio na sua produção, resultando no aumento de sua concentração na corrente sanguínea. Este fato contribui para a deposição de colesterol nas artérias, a formação de plaquetas e o desenvolvimento de doenças cardiovasculares.

Reforça-se desta forma, a necessidade de se divulgarem informações acerca dos teores de colesterol em alimentos. Com este intuito, pesquisas foram publicadas no exterior (ÍMRE; SAGLIK, 1998; KARAKOLTSIDIS; ZOTOS;
CONSTANTINIDES, 1995; MATHEW et al., 1999). No Brasil, entre outros estudos, foram divulgados os teores de colesterol em carnes, ovos e massas com ovos (BRAGAGNOLO, 1992), carnes de bode (ALMEIDA et al., 1997) e peru (BAGGIO; VICENTE; BRAGAGNOLO, 2002), camarões de água doce e marinha (BRAGAGNOLO; RODRIGUEZ-AMAYA, 1997; 2001), peixes de água doce e marinha (MOREIRA et al., 2001; MOURA; TENUTA-FILHO, 2002) e ovos de galinha e codorna (BRAGAGNOLO; RODRIGUEZ-AMAYA, 2003).

Outro aspecto importante relativo à presença de colesterol em alimentos está no fato comprovado de que o colesterol pode sofrer oxidação, formando óxidos de colesterol (AL-SAGHIR et al., 2004; ECHARTE; ZULET; ASTIASARAN, 2003; MOURA; TENUTA-FILHO, 2002; OSADA et al., 1993; PIE; SPAHIS; SEILLAN, 1990). Estes óxidos são conside-

1 Universidade Federal do Ceará - UFC

2 Centro de Ciências Agrárias, Laboratório de Recursos Aquáticos - LARAQ, Departamento de Engenharia de Pesca, Universidade Federal do Ceará - UFC, Campus do Pici, CEP 60356-000, Fortaleza-CE, Brasil, E-mail: maiadep@ufc.br

${ }^{*}$ A quem a correspondência deve ser enviada 
rados agentes aterogênicos, carcinogênicos e mutagênicos (SHOZEN et al., 1995).

Com o intuito de contribuir para a divulgação de informações acerca do teor de colesterol em alimentos brasileiros, realizou-se a presente pesquisa, com amostras de peixes marinhos e de água doce comercializados na cidade de Fortaleza, estado do Ceará.

\section{Material e métodos}

\subsection{Amostras}

Todas as amostras investigadas foram de peixes comercializados na cidade de Fortaleza, estado do Ceará, adquiridas em 2002. O peixe marinho constou de filés congelados de pargo, Lutjanus purpureus. O número de amostras (n) perfez 36, coletados de janeiro a dezembro, com pesos dos filés congelados variando de 400 a $500 \mathrm{~g}$. As três espécies de peixes de água doce, todas mantidas em gelo durante sua comercialização, constaram de: tilápia do Nilo, Oreochromis niloticus, $\mathrm{n}=60$, período de janeiro a dezembro, com pesos dos peixes inteiros variando entre 500 e 700 g; curimatã, Prochilodus cearensis, $\mathrm{n}=15$, período de janeiro a maio, com os pesos dos peixes inteiros variando de 700 a 1000 g, e sardinha inteira, Triportheus angulatus, $\mathrm{n}=90$, período de janeiro a maio, com peso médio em torno de $40 \mathrm{~g}$.

\subsection{Preparação de amostras para análise}

Foi analisada a parte comestível dos peixes, ou seja, os filés sem pele e isentos de espinhas intramusculares. Estas foram homogeneizadas em processador/liquidificador Mallory Triter, antes da realização das determinações químicas descritas a seguir.

\subsection{Determinações químicas}

\section{Colesterol}

O teor do colesterol foi determinado pelo método colorimétrico descrito por Bohac et al. (1988), utilizando-se a matéria insaponificável recuperada de lipídio total (50-200 mg), extraído pelo método de Bligh e Dyer (1959). O teor de colesterol das amostras foi medido em triplicata, usando-se curva padrão (SIGMA, pureza > 99\%) na faixa de 0 a $250 \mu \mathrm{g} .5 \mathrm{~mL}^{-1}$ e leitura da absorbância em comprimento de onda de $490 \mathrm{~nm}$ utilizando-se espectrofotômetro UV/VIS SHIMADZU, modelo UV-1601.

\section{Umidade}

O teor de umidade presente nas amostras foi determinado por secagem em estufa a $105 \pm 5{ }^{\circ} \mathrm{C}$, seguindo-se as recomendações descritas por Nagakura (1972).

\section{Proteina total (PT)}

O teor de proteína total foi medido por meio do método semi-micro Kjeldahl, utilizando-se o fator de 6,25 para conversão de nitrogênio total em proteína total de acordo com Pearson (1973).

\section{Lipídio total (LT)}

Para a determinação do teor de lipídio total, foi usado o método de extração a frio de Bligh e Dyer (1959).

\section{Cinzas (CZ)}

A porcentagem de cinzas nas amostras foi determinada conforme o método de Nagakura (1972), por meio de incineração em forno mufla na temperatura de $550{ }^{\circ} \mathrm{C}$.

\section{Carboidrato (CHO)}

O percentual de carboidrato nas amostras foi obtido por diferença $[\% \mathrm{CHO}=100-(\%$ água $+\% \mathrm{PT}+\% \mathrm{LT}+\% \mathrm{CZ})]$, conforme descrito por Brasil/ANVISA (2001).

\section{Valor energético (VE)}

Utilizando-se os fatores de conversão de Atwater de 4,0 kcal.g $\mathrm{g}^{-1}$ para proteína e carboidrato e de 9,0 kcal.g ${ }^{-1}$ para gordura, empregou-se a expressão abaixo para o cálculo de VE (BRASIL/ANVISA, 2001)(Equação 1):

VE $\left(\mathrm{kcal} .100 \mathrm{~g}^{-1}\right)=\% \mathrm{LT} \times 9 \mathrm{kcal} . \mathrm{g}^{-1}+\% \mathrm{PT} \times 4 \mathrm{kcal} . \mathrm{g}^{-1}+$ $\% \mathrm{CHO} \times 4 \mathrm{kcal}^{-\mathrm{g}^{-1}}$

\subsection{Análise estatística}

Os parâmetros estatísticos - compreendendo a análise de variância, o teste de Tukey e a igualdade de médias - foram aplicados aos dados de colesterol, de composição química e de valor energético, segundo Centeno (1999) e Montgomery (1976).

\section{Resultados e discussão}

\subsection{Teores de colesterol e lipídio total}

Os teores de Colesterol (COL) e Lipídio Total (LT) encontrados nos músculos das espécies de pargo, tilápia, curimatã e sardinha, provenientes do estado do Ceará, estão apresentados na Tabela 1.

O pargo, cujo teor médio de colesterol foi de 33,5 \pm 6,9 mg. $100 \mathrm{~g}^{-1}$ de amostra (valores mínimo e máximo: 23,7 - 46,7), apresentou variação regular ao longo dos 12 meses de análises - Coeficiente de Variação $(\mathrm{CV})=20,6 \%$ - , mas sem apresentar correlação com o teor de lipídio total, com coeficiente de correlação $(r)=-0,15039$. Vila Nova et al. (2005) relataram

Tabela 1. Teor de colesterol e lipídio total encontrado em filés de peixes do estado do Ceará.

\begin{tabular}{lcc}
\hline \multicolumn{1}{c}{ Amostras } & $\begin{array}{c}\text { Colesterol } \\
\left(\mathrm{mg} .100 \mathrm{~g}^{-1}\right) \\
(\text { média } \pm \mathrm{dp})^{1,2}\end{array}$ & $\begin{array}{c}\text { Lipídio total }(\%) \\
(\text { média } \pm \mathrm{dp})\end{array}$ \\
\hline Pargo (peixe marinho) & $33,5 \pm 6,9^{\mathrm{a}}$ & $1,0 \pm 0,5^{\mathrm{a}}$ \\
Tilápia (peixe de água doce) & $28,4 \pm 8,1^{\mathrm{a}}$ & $1,2 \pm 0,8^{\mathrm{ab}}$ \\
Curimatã (peixe de água doce) & $93,8 \pm 26,0^{\mathrm{b}}$ & $3,2 \pm 1,2^{\mathrm{bc}}$ \\
Sardinha (peixe de água doce) & $61,2 \pm 4,7^{\mathrm{bc}}$ & $4,6 \pm 0,4^{\mathrm{c}}$ \\
\hline
\end{tabular}

${ }^{1} \mathrm{dp}=$ desvio padrão; $\mathrm{e}^{2}$ valores nas colunas marcados com as mesmas letras não diferem significativamente $(\mathrm{p}<0,05)$. 
para o pargo nordestino uma média de 12,75 $\pm 0,29 \mathrm{mg} \cdot 100 \mathrm{~g} \mathrm{~g}^{-1}$ $(\mathrm{CV}=2,2 \%)$ de colesterol quantificado por CLAE, mas para "snapper" da Flórida - USA, foi relatado um teor médio de $45 \mathrm{mg} .100 \mathrm{~g}^{-1}$ (SEAFOOD FACTS SHEET, 2006).

$A$ tilápia, com $28,4 \pm 8,1 \mathrm{mg} \cdot 100 \mathrm{~g} \mathrm{~g}^{-1}(20,0-46,6)$, foi o peixe que teve o menor teor de colesterol, mas por outro lado, foi a espécie que teve a maior amplitude de valores $(\mathrm{CV}=28,5 \%)$ durante o período de 12 meses de investigação. Porém, diferentemente do pargo, houve uma razoável correlação diretamente proporcional $(r=0,79699)$ entre o teor de LT e COL durante o período de analise. Valores inferiores foram registrados por Vila Nova et al. (2005), que notaram diferenças significativas entre a tilápia natural $\left(10 \mathrm{mg} .100 \mathrm{~g}^{-1}\right)$ em relação àquela adaptada em água salina $\left(8,2 \mathrm{mg} .100 \mathrm{~g}^{-1}\right)$ e àquela sexualmente revertida ( $\left.8,7 \mathrm{mg} \cdot 100 \mathrm{~g}^{-1}\right)$. Todavia, valores superiores foram relatados para a tilápia, Oreochromis spp., comercializada na capital de São Paulo (69,1 mg.100 g ${ }^{-1}$ (LUZIA et al., 2003), para a Tilapia mozambique, da Arábia $(53,9 \pm 1,9$ mg.100 g-1) (EWAIDAH, 1993), e para a Tilápia, Oreochromis mossambica, da Índia (41,8 mg.100 g ${ }^{-1}$ ) (MATHEW et al., 1999).

O curimatã, Prochilodus cearensis, foi a espécie que apresentou o maior teor de colesterol, com média de 93,8 $\pm 26,0$ mg.100 g-1 (59,4-122,3; CV =27,7\%), e também uma forte correlação inversa com o teor de LT $(r=-0,96263)$ durante os cinco meses de análises. Para o curimbatá, Prochilodus spp. de São Paulo, Luzia et al. (2003) encontraram um teor médio de $82,2 \mathrm{mg} .100 \mathrm{~g}^{-1}$, razoavelmente próximo do teor do curimatã cearense.

A sardinha cearense de água doce, Triportheus angulatus, foi a segunda espécie de peixe que apresentou o maior teor de colesterol, com 61,2 $\pm 4,7 \mathrm{mg} .100 \mathrm{~g}^{-1}(56,2-66,1)$, com pouca variação durante os cinco meses de investigação $(\mathrm{CV}=7,7 \%)$. Similarmente ao pargo, para a sardinha também não houve correlação $(r=-0,09552)$ entre os teores de COL e LT. No Brasil, foi relatado um teor médio de 79,5 mg.100 g $\mathrm{g}^{-1}$ para a sardinha marinha, Sardinella spp. (LUZIA et al., 2003), enquanto no âmbito internacional foram descritos teores de $43,4 \mathrm{mg} \cdot 100 \mathrm{~g} \mathrm{~g}^{-1}$ na sardinha marinha, Sardina pilchardus, de $63 \pm 1,1 \mathrm{mg} .100 \mathrm{~g} \mathrm{~g}^{-1}$ para a sardinha, Clupea pilchardus, do Mediterrâneo (KARAKOLTSIDIS; ZOTOS; CONSTANTINIDES, 1995), e média de $62,4 \mathrm{mg} .100 \mathrm{~g}^{-1}$ para três espécies de sardinhas marinhas da Índia (MATHEW et al., 1999).

A análise estatística mostrou que houve diferença significativa $(\mathrm{p}<0,05)$ entre as médias de colesterol no músculo do pargo (peixe marinho) em relação aos peixes de água doce sardinha e curimatã, que apresentaram, respectivamente, cerca de duas e três vezes maior concentração. Todavia, não se pode generalizar que os peixes de água doce apresentam concentração de colesterol mais elevada do que o peixe marinho, porque este teve menor teor de colesterol do que a tilápia, sem apresentar diferença significativa $(p>0,05)$. Todos os peixes apresentaram teores de colesterol inferiores aos teores dos camarões, sendo que, entre estes, também não foi possível generalizar a existência de diferenças entre as espécies marinhas e as espécies de água doce (BRAGAGNOLO; RODRIGUEZ-AMAYA, 1997; 2001).
O teor médio de colesterol presente nas quatro amostras do estado do Ceará foi de $54,2 \mathrm{mg} .100 \mathrm{~g}^{-1}$ de amostra, valor próximo daquele obtido para onze espécies de peixes do Golfo da Arábia (65 mg.100 g-1), onde, com algumas exceções, também foi notada a existência de diferenças no teor de colesterol entre as espécies (EWAIDAH, 1993). Para Ímre e Saglik (1998), pequenas diferenças desse composto foram encontradas durante a análise de cinco espécies de peixes da Turquia, que apresentaram média de $52 \mathrm{mg} .100 \mathrm{~g}^{-1}$ de colesterol.

O teor de lipídio total (LT) para o pargo (1,0 0 0,5\%) está próximo dos descritos por Oliveira (1999) e Vila Nova et al. (2005), que relataram médias de $1,9 \%$ e $1,18 \pm 0,01 \%(\mathrm{CV}=0,85 \%)$ de lipídios musculares, respectivamente. Por outro lado, Nunes et al. (1976) encontraram uma média de 2,1\% (1,3-3,7\%) e diferenças estatísticas significativas entre os meses analisados, enquanto Sales e Monteiro (1988) não comentaram tal ocorrência para o pargo, que teve uma média de 2,4\% (1,7-3,7\%).

A tilápia também apresentou baixo teor de lipídio total, com média de 1,2\%. Esta média foi inferior àquela de 1,6\% encontrada no músculo da tilápia cultivada, Oreochromis spp. (LUZIA et al., 2003), mas superior aos valores relatados por Vila Nova et al. (2005). Na Venezuela, Elisabetta et al. (2001) descreveram para o híbrido de tilápia (O.mossabicus $\times$ O.urolepis $\mathrm{X}$ O.hornorum $\times$ O.niloticus $\times$ O.aureus) um teor médio (1,0\%), próximo do encontrado no presente trabalho.

O filé de curimatã, com $3,2 \pm 1,2 \%(1,5-4,3 \%, \mathrm{CV}=37,5 \%)$, apresentou, entre os peixes, o segundo teor médio de gordura mais elevado. Dados de literatura registram para esta mesma espécie valores médios de 2,9 $\pm 1,3 \%$ (OLIVEIRA, 1999) até 6,6\% (4,8 - 8,5\%) (SALES; SALES, 1990). Na capital de São Paulo, o curimbatá, Prochilodus spp., teve média de $8,2 \%$ (9,70\% no verão e 6,67\% no inverno) (LUZIA et al., 2003).

O maior teor de LT foi encontrado no filé de sardinha, com 4,6\% (4,1-5,0\%), valor igual ao relatado por Sales e Sales (1990), em cujo estudo esta espécie apresentou variação entre 1,6 a $7,7 \%$.

Os teores de lipídio total das quatro espécies investigadas, que ficaram compreendidos entre 1,0 e $4,6 \%$, permitem classificá-las como espécies magras, segundo o critério estabelecido por Stansby (1962), que preconiza um teor inferior a 5\%.

\subsection{Composição centesimal e valor energético}

Os dados relativos aos teores de umidade, proteína total, lipídio total cinza, carboidratos (por diferença) e o valor energético ou calórico das amostras de pargo, tilápia, curimatã e sardinha estão apresentados na Tabela 2.

Verifica-se que o maior teor de umidade foi obtido para o pargo $(80,7 \%)$, valor este muito próximo daquele obtido para a tilápia $(80,2 \%)$. A sardinha com $77,2 \%$ e o curimatã com $76,4 \%$ apresentaram valores próximos entre si. Uma diferença significativa ocorreu entre o teor de umidade do curimatã em relação ao pargo e à tilápia, mas não em relação à sardinha. Estes dados estão em concordância com os valores divulgados por diversos autores (GURGEL; FREITAS, 1972; NUNES et al., 1976; 
OGAWA, 1999; OLIVEIRA, 1999; PUWASTIEN et al., 1999; SALES; MONTEIRO, 1988; TORRES et al., 2000).

Segundo Ogawa (1999), a composição protéica da carne de peixe pode variar em função da espécie, do tamanho, do sexo e da época do ano; porém, geralmente, o músculo contém cerca de $20 \%$ de proteína. Nota-se pelos dados na Tabela 2 que os teores de proteína nas quatro espécies ficaram compreendidos na faixa de 17,6 a $18,7 \%$, confirmando os resultados publicados pela literatura brasileira e internacional (ELISABETTA et al., 2001; GURGEL; FREITAS, 1972; NUNES et al., 1976; OLIVEIRA, 1999; SALES; MONTEIRO, 1988; TORRES et al., 2000). Em termos de média, o curimatã foi a espécie que teve o maior teor de proteína $(18,7 \%)$, seguido pelo pargo $(18,4 \%)$, tilápia $(17,7 \%)$ e sardinha $(17,6 \%)$. Não houve, contudo, diferenças significativas $(p>0,05)$ entre elas.

Os teores médios de cinza nas diversas amostras de pescado do estado do Ceará também não apresentaram diferenças significativas $(\mathrm{p}>0,05)$ entre as espécies. Além disso, todos os valores apresentaram conformidade com os trabalhos publicados para pescado que, segundo Stansby (1962), devem variar entre 0,4 e $1,5 \%$

Os teores de carboidratos variaram na faixa de 0,3 a 2,0\% nas espécies investigadas, estando, assim, razoavelmente próximos dos valores de 0,3 a 1,0\% descritos por Ogawa (1999), para peixes em geral. Para Karakoltsidis et al. (1995), em cinco espécies de peixes, os teores de carboidrato (por diferença) variaram de 0,3 a $6 \%$. Na medida em que a percentagem de carboidrato foi obtida por diferença, não foi aplicada a análise estatística. Entretanto, estes dados foram usados no cálculo do valor energético das amostras.

Os valores energéticos médios nos músculos de pargo $\left(83,6 \mathrm{kcal} .100 \mathrm{~g}^{-1}\right)$ e de tilápia $\left(83,7 \mathrm{kcal} .100 \mathrm{~g}^{-1}\right)$ foram inferiores aos valores do curimatã $\left(108,4 \mathrm{kcal} .100 \mathrm{~g}^{-1}\right)$ e da sardinha $\left(111,8 \mathrm{kcal} .100 \mathrm{~g}^{-1}\right)$. Nota-se que as duas primeiras espécies não diferiram entre si $(p>0,05)$ e que o mesmo ocorre entre as duas últimas. Estas, por sua vez, tiveram valores calóricos diferentes quando comparados com as duas primeiras espécies (Tabela 2). Pesquisa publicada por Gurgel e Freitas (1972) mostrou que existiu uma variação de 104 a $293 \mathrm{kcal} .100 \mathrm{~g}^{-1}$ de amostra, em 12 espécies de peixes de água doce do Ceará, incluindo-se o curimatã comum (183 kcal), a tilápia (116 kcal) e a sardinha ( $120 \mathrm{kcal})$. Estes dados confirmam que os peixes podem ser considerados alimentos de baixo valor calórico quando comparados com outros alimentos, tais como doce de leite ( $316 \mathrm{kcal})$, manteiga ( $740 \mathrm{kcal})$, queijo de Minas (335 kcal), ovo de codorna (135 kcal), ovo de galinha (146 kcal), coxa de frango (156 kcal), contrafilé bovino (192 kcal) e lombo de porco (212 kcal) (TORRES et al., 2000).

\section{Conclusões}

O curimatã e a sardinha apresentaram teores elevados de colesterol e estatisticamente diferentes do pargo, uma espécie de água marinha. Entre o pargo e a tilápia (peixe de água doce), não houve diferença significativa no teor de colesterol.

Todas as amostras de peixes pesquisadas podem ser consideradas pobres em colesterol, quando os valores encontrados são comparados com os resultados publicados para outros alimentos de origem animal.

Quanto à composição centesimal, foi o lipídio total que apresentou a maior variabilidade ao longo dos meses investigados. Contudo, na medida em que apresentaram, de forma constante, baixos valores, as espécies estudadas podem ser consideradas magras.

Uma forte correlação inversamente proporcional ocorreu entre o teor de colesterol e o teor de gordura do curimatã. Diferentemente, na tilápia, a correlação foi positiva e mediana.

Não foram observadas diferenças significativas nos teores de proteína e cinza entre os peixes de água doce e o peixe marinho.

Os dados relativos aos teores de gordura e de colesterol e ao valor energético mostram uma tendência de valores superiores para os peixes de água doce em relação ao peixe marinho.

\section{Referências bibliográficas}

ALMEIDA, M. M. M. et al. Cholesterol and phospholipids levels in goat meat as affected by dietary calcium. Pesquisa Agropecuária Brasileira, v. 32, n. 5, p. 555-558, 1997.

AL-SAGHIR, S. et al. Effects of Different Cooking Procedures on Lipid Quality and Cholesterol Oxidation of Farmed Salmon Fish (Salmo salar). Journal of Agricultural and Food Chemistry, v. 52, n. 16, p. 5290-5296, 2004.

BAGGIO, S. R.; VICENTE, E.; BRAGAGNOLO, N. Cholesterol Oxides, Cholesterol, Total Lipid, and Fatty Acid Composition in Turkey Meat. Journal of Agricultural and Food Chemistry, v. 50, n. 21, p. 5981-5986, 2002.

BLIGH, E. G.; DYER, W. A rapid method of total lipid extraction and purification. Canadian Journal of Biochemistry and Physiology, v. 37, n. 8, p. 911-917, 1959.

BOHAC, C. R. et al. Assessment of methodologies for colorimetric cholesterol assay of meats. Journal of Food Science, v.53, n. 6, p. 1642-1644, 1988.

BRAGAGNOLO, N. Determinação dos teores de colesterol em carnes, ovos e massas com ovos. Campinas, 1992. 105 p. Dissertação (Mestrado em Engenharia de Alimentos), Universidade Estadual de Campinas.

Tabela 2. Composição centesimal e valor energético em filés de peixes do estado do Ceará.

\begin{tabular}{|c|c|c|c|c|c|c|}
\hline \multirow[t]{2}{*}{ Amostras } & \multicolumn{5}{|c|}{ Composição Centesimal (\%) (média \pm desvio padrão) } & \multirow{2}{*}{$\begin{array}{l}\text { Valor energético } \\
\left(\mathrm{kcal} .100 \mathrm{~g}^{-1}\right)\end{array}$} \\
\hline & Umidade & Proteína & Lipídio total & Cinza & Carboidrato & \\
\hline Pargo & $80,7 \pm 1,4^{\mathrm{a}}$ & $18,4 \pm 1,5^{\mathrm{a}}$ & $1,0 \pm 0,5^{\mathrm{a}}$ & $0,7 \pm 0,2^{\mathrm{a}}$ & $0,5 \pm 0,3$ & $83,6 \pm 7,4^{\mathrm{a}}$ \\
\hline Tilápia & $80,2 \pm 1,7^{\mathrm{ab}}$ & $17,7 \pm 0,8^{\mathrm{a}}$ & $1,2 \pm 0,8^{\mathrm{ab}}$ & $0,8 \pm 0,3^{\mathrm{a}}$ & $0,6 \pm 0,2$ & $83,7 \pm 8,8^{\mathrm{a}}$ \\
\hline Curimatã & $76,4 \pm 1,2^{c}$ & $18,7 \pm 1,6^{\mathrm{a}}$ & $3,2 \pm 1,2^{\mathrm{bc}}$ & $0,8 \pm 0,3^{\mathrm{a}}$ & $2,0 \pm 2,3$ & $108,4 \pm 11,2^{\mathrm{b}}$ \\
\hline Sardinha & $77,2 \pm 0,7^{\mathrm{abc}}$ & $17,6 \pm 1,0^{\mathrm{a}}$ & $4,6 \pm 0,4^{\mathrm{c}}$ & $1,0 \pm 0,4^{\mathrm{a}}$ & $0,3 \pm 0,0$ & $111,8 \pm 4,7^{\mathrm{b}}$ \\
\hline
\end{tabular}

${ }^{1}$ Valores nas colunas marcados com as mesmas letras não diferem significativamente $(p<0,05)$. 
BRAGAGNOLO, N.; RODRIGUEZ-AMAYA, D. Otimização da determinação de colesterol por CLAE e teores de colesterol, lipídio total e ácidos graxos em camarão rosa (Penaeus brasiliensis). Ciência e Tecnologia de Alimentos, v. 17, n. 3, p. 275-280, 1997.

BRAGAGNOLO, N.; RODRIGUEZ-AMAYA, D. B. Total lipid, cholesterol, and fatty acids of farmed freshwater prawn (Macrobrachium rosenbergii) and wild marine shrimp (Penaeus brasiliensis, Penaeus schimitti, Xiphopenaeus kroyeri). Journal of Food Composition and Analysis, v. 14, n. 4, p. 359-369, 2001.

.Comparison of the cholesterol content of Brazilian chicken and quail eggs. Journal of Food Composition and Analysis, v. 16, n. 2, p. 147-153, 2003.

BRASIL. ANVISA. AGÊNCIA NACIONAL DE VIGILÂNCIA SANITÁRIA. Resolução - RDC no 40, de 21 de março de 2001. Regulamento técnico para rotulagem nutricional de alimentos e bebidas embaladas. Brasília, 2001.

CENTENO, A. J. Curso de estatística aplicada à biologia. 2 ed. Goiânia: Ed. da UFB, 1999. 234 p.

CORMILLOT, A. O colesterol: O que é, quando prejudica a saúde e o que fazer para controlá-lo. São Paulo: Editora Caras, 2002. 191 p.

ECHARTE, M.; ZULET, M.; ASTIASARAN, I. Oxidation process affecting fatty acids and cholesterol in fried and roasted salmon. Journal of Agricultural and Food Chemistry, v. 49, n. 11, p. 5662-5667, 2001.

ELISABETTA, T. et al. Efecto del tiempo de retardo en la refrigeración sobre la frescura de la Tilapia (Oreochromis spp.) cultivada. Anales Venezolanos de Nutrición, v. 14, n. 1, p. 3-8, 2001.

EWAIDAH, E. H. Cholesterol, fat and food energy content of selected raw and cooked commercial fish species from the Arabian Gulf. Ecology of Food and Nutrition, v. 30, p. 283-292, 1993.

GURGEL, J. J. S.; FREITAS, J. V. F. Sobre a composição química de doze espécies de peixes de valor comercial dos açudes do Nordeste brasileiro. Boletim Técnico do DNOCS, v. 30, n. 1, p. 45-57, 1972.

ÍMRE, S.; SAGLIK, S. Fatty acid composition and cholesterol content of some Turkish fish species. Turkish Journal of Chemistry, v. 22, n. 4, p. 321-324, 1998.

KARAKOLTSIDIS, P. A.; ZOTOS, A.; CONSTANTINIDES, S. M. Composition of the comercially important Mediterranean finfish, crustaceans, and molluscs. Journal of Food Composition and Analysis, v. 8, n. 3, p. 258-273, 1995.

LUZIA, L. A. et al. The influence of season on the lipid profiles of five commercially important species of Brazilian fish. Food Chemistry, v. 83 , n. 1, p. 93-97, 2003.

MATHEW, S. et al. Cholesterol content of Indian fish and shellfish. Food Chemistry, v. 66, n. 4, p. 455-461, 1999.

MONTGOMERY, D. C. Design and analysis of experiments. New York: Institute of Technology, 1976. 418 p.

MOREIRA, A. B. et al. Fatty acids profile and cholesterol contents of three Brazilian Brycon freshwater fishes. Journal of Food Composition and Analysis, v. 14, n. 6, p. 565-570, 2001.
MOURA, A. F. P.; TENUTA-FILHO, A. Effects of processing on free cholesterol and 7-ketocholesterol concentrations in pink-shrimp. Ciência e Tecnologia de Alimentos, v. 22, n. 2, p. 117-121, 2002.

NAGAKURA, K. General analysis. In: OKADA, M. (Ed.). Utilization of marine products. Tokyo: Overseas Technical Cooperation Agency, 1972. p. 159-169.

NUNES, M. L. et al. Composição química de alguns peixes marinhos de Nordeste brasileiro. Arquivos de Ciências do Mar, v. 16, n. 1, p. 23-26, 1976.

OGAWA, M. Química do pescado. In: MASAYOSHI, M.; MAIA, E.L. (Eds.). Manual de pesca - ciência e tecnologia do pescado. São Paulo: Varela, 1999. cap. 4, p. 29-71.

OLIVEIRA, S. L. C. L. Estudo dos constituintes lipídicos em peixes do Ceará. Ceará, 1999. 117p. Dissertação - (Mestrado em Engenharia de Pesca), Departamento de Engenharia de Pesca, Universidade Federal do Ceará.

OSADA, K. et al. Levels and formation of oxidized cholesterols in processed marine foods. Journal of Agricultural and Food Chemistry, v. 41, n. 11, p. 1893-1898, 1993.

PEARSON, D. General methods - basic constituintes. In: Laboratory techniques in food analysis. New York: John Wiley \& Sons, 1973. cap. 2, p. 27-77.

PIE, J. E.; SPAHIS, K.; SEILLAN, C. Evaluation of oxidative degradation of cholesterol in food and food ingredients: Identification and quantification of cholesterol oxides. Journal of Agricultural and Food Chemistry, v. 38, n. 4, p. 973-979, 1990.

PUWASTIEN, P. et al. Proximate composition of raw and cooked Thai freshwater and marine fish. Journal of Food Composition and Analysis, v. 12, n. 1, p. 9-16, 1999.

SALES, R. O.; MONTEIRO, J. C. S. Estudo da composição química e rendimento de quatro espécies marinhas de interesse comercial. Ciência Agronômica, v. 19, n. 1, p. 43-47, 1988.

SALES, R. O.; SALES, A. M. Estudo da composição química e rendimento de dez espécies de pescado de água doce de interesse comercial nos açudes do Nordeste Brasileiro. Ciência Agronômica., v. 21, n. 1/2, p. 27-30, 1990.

SEAFOOD FACTS SHEET. Nutrient values for Florida seafood. Florida Seafood Health Facts. Disponível no site: <http://app2.fl-seafood. com/pubs/pubform/pdf/English_Brochure_Florida_Seafood_ Healthy_Facts.pdf

SHOZEN, K. J. et al. Formation of colesterol oxides in marine fish products induced by grilling. Fisheries Science, v. 61, n. 5, p. 818-821, 1995.

STANSBY, M. E. Proximate composition of fish. In: HEEN, E.; KREUZER, R. (Ed.). Fish in nutrition. London: Fishing News, 1962. p. 55-60.

TORRES, E. A. F. S. et al. Composição centesimal e valor calórico de alimentos de origem animal. Ciência e Tecnologia de Alimentos, v. 20 , n. 2 , p. $145-150,2000$.

VILA NOVA, C. M. V. M.; GODOY, H. T.; ALDRIGUE, M. L. Chemical composition, cholesterol content and characterization of total lipids of Nile tilapia (Oreochromis niloticus) and pargo (Lutjanus purpureus). Ciência eTecnologia de Alimentos, v. 25, n. 3, p. 430-436, 2005. 